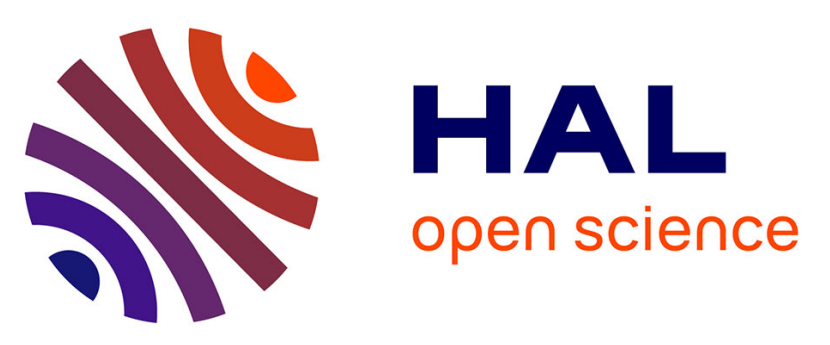

\title{
Influence of the ultra-slow nucleation and growth dynamics on the room-temperature hysteresis of spin-crossover single crystals
}

Volodymyr M Hiiuk, Karl Ridier, Il'ya A Gural'Skiy, Alexander A Golub, Igor O Fritsky, Gábor Molnár, William Nicolazzi, Azzedine Bousseksou

\section{To cite this version:}

Volodymyr M Hiiuk, Karl Ridier, Il'ya A Gural'Skiy, Alexander A Golub, Igor O Fritsky, et al.. Influence of the ultra-slow nucleation and growth dynamics on the room-temperature hysteresis of spin-crossover single crystals. Chemical Physics Letters, 2021, 770, pp.138442. 10.1016/j.cplett.2021.138442 . hal-03179259

\section{HAL Id: hal-03179259 \\ https://hal.science/hal-03179259}

Submitted on 24 Mar 2021

HAL is a multi-disciplinary open access archive for the deposit and dissemination of scientific research documents, whether they are published or not. The documents may come from teaching and research institutions in France or abroad, or from public or private research centers.
L'archive ouverte pluridisciplinaire HAL, est destinée au dépôt et à la diffusion de documents scientifiques de niveau recherche, publiés ou non, émanant des établissements d'enseignement et de recherche français ou étrangers, des laboratoires publics ou privés. 


\title{
Influence of the ultra-slow nucleation and growth dynamics on the room-temperature hysteresis of spin-crossover single crystals
}

\author{
Volodymyr M. Hiiuk ${ }^{1,2,3}$, Karl Ridier ${ }^{4}$, Il'ya A. Gural'skiy $^{1,2 *}$, Alexander A. Golub ${ }^{3}$, \\ Igor O. Fritsky ${ }^{1,2}$, Gábor Molnár ${ }^{4}$, William Nicolazzi ${ }^{4}$, Azzedine Bousseksou ${ }^{4 * *}$
}

\footnotetext{
${ }^{1}$ Department of Chemistry, Taras Shevchenko National University of Kyiv, 64 Volodymyrska St., 01601 Kyiv, Ukraine.

${ }^{2}$ UkrOrgSyntez Ltd., 67 Chervonotkatska St., 02094 Kyiv, Ukraine.

${ }^{3}$ Faculty of Natural Sciences, National University of Kyiv-Mohyla Academy, 2 Skovorody St., 04070 Kyiv, Ukraine.
}

${ }^{4}$ Laboratoire de Chimie de Coordination, CNRS \& Université de Toulouse, 205 route de Narbonne, 31077 Toulouse, France.

\begin{abstract}
Nucleation and growth phenomena associated with the thermally-induced spin transition was studied using optical microscopy in single crystals of a cyanoheterometallic coordination polymer $\left[\mathrm{Fe}(1,6 \text {-naphthyridine })_{2}\left(\mathrm{Ag}(\mathrm{CN})_{2}\right)_{2}\right]$. Despite the fact that the spin-crossover phenomenon occurs near room temperature ( $c a$. 290-300 K), the experimental results show the existence of significant kinetic effects on the hysteretic behaviour of the spin transition in this compound. Ultra-slow switching rates and phase-boundary propagation velocities were observed (down to $3 \mathrm{~nm} \mathrm{~s}^{-1}$ ), several orders of magnitude lower than customary. Our observations indicate that these peculiar spin-crossover properties (slow switching kinetics, scan-rate-dependent transition temperatures) are presumably related to the presence of numerous microstructural defects in the crystals, which is an intrinsic characteristic of this compound.
\end{abstract}

Keywords: Spin crossover; Nucleation and growth dynamics of the phase; Hysteresis; Defects; Single crystals.

\section{Corresponding author:}

*II'ya A. Gural'skiy: illia.guralskyi@gmail.com

**Azzedine Bousseksou: azzedine.bousseksou@lcc-toulouse.fr 


\section{Introduction}

Iron (II) spin-crossover (SCO) complexes constitute one of the most interesting representatives of switchable molecular materials [1-3]. The SCO phenomenon in these coordination complexes is related to the ability of the central metal ion to change its spin state, in a reversible manner, under the influence of external stimuli [4]. These complexes, which have the particularity of exhibiting bistability properties, characterized by the possible emergence of hysteretic phenomena at technologically relevant temperatures, constitute appealing molecular systems for practical applications, for example, as active elements of information processing, energy transducing and display devices [5].

Due to the drastic change of different physical (magnetic, optical, mechanical, electrical, etc.) properties during the SCO, this phenomenon can be monitored by various techniques (magnetometry, X-ray diffraction, Mössbauer spectroscopy, differential scanning calorimetry, optical microscopy, etc.) [4]. Among these techniques, optical microscopy (OM) occupies a special place because it allows a real-time, non-destructive and non-contact detection of the SCO in individual single crystals, combining high temporal and spatial resolutions. (N.B. Here we primarily refer to $\mathrm{OM}$ in bright-field, transmission mode, which probes local absorbance changes and allows for quantitative spatiotemporal studies of the spin-state transformation. It should be noted, however, that $\mathrm{OM}$ has been also used to probe refractive index and luminescence intensity changes, enabling to detect the spin transition in single SCO micro-nano-particles [6-8]). OM studies on large single crystals (typically 50-100 $\mu \mathrm{m}$ ) help to better understand the fundamental aspects of the spin-state switching process (nucleation and growth phenomenon, spatiotemporal properties), which have an influence on the key characteristics of the spin transition (switching temperatures, sharpness, completeness and hysteretic behaviour).

The first (visible-range) OM studies on SCO single crystals were performed by Jeftic and Hauser [9]. These authors observed the spatiotemporal development of the high-spin (HS) to low-spin (LS) relaxation in a single crystal of the SCO complex $\left[\mathrm{Fe}(\mathrm{ptz})_{6}\right]\left(\mathrm{BF}_{4}\right)_{2}(\mathrm{ptz}=1$-propyltetrazole). The observed inhomogeneous features were explained due to structural modifications and internal stresses in the crystal associated with the spin-state transition. In the past decade, numerous OM studies on different SCO single crystals allowed significant advances in the understanding of the spatiotemporal properties of the spin transition with the identification of some general features, which have been reviewed by different groups involved in this research [10, 11]. In summary, in highly cooperative compounds exhibiting hysteretic behaviour, heterogeneous nucleation 
followed by the formation of a well-defined HS/LS interface and its slow propagation (typically between 1-10 $\mu \mathrm{m} \mathrm{s}^{-1}$, but sometimes up to a few hundreds of $\mu \mathrm{m} \mathrm{s}^{-1}$ ) was observed in both cooling and heating modes. Moreover, it was shown that the nucleation and growth phenomenon significantly depends on the crystal morphology, the local microstructure and the presence of internal defects. Several studies have indeed pointed out the prominent role of crystal edges and defects on the nucleation process and the phase boundary propagation $[12,13]$, while the possibility of modulating the spatiotemporal dynamics of the nucleation and growth phenomenon by the creation of artificial micrometric defects has been experimentally demonstrated [14]. Another general observation is that the SCO is often accompanied by irreversible damage (cracks, fractures, dislocations, etc.) upon subsequent switching cycles, which mainly originate from the internal stresses generated by the transition process - due to the relatively large volume change (and lattice mismatch) between the HS and LS phases. Overall, the substantial volume change in SCO solids appears as a key parameter at the origin of the slow phase-boundary propagation velocity and the rather low mechanical resilience of SCO crystals [11].

In the present work, we report on OM investigations of SCO single crystals of [Fe(1,6naphthyridine $)_{2}\left(\operatorname{Ag}(\mathrm{CN})_{2}\right)_{2}$ ] (1). This two-dimensional (2D) coordination network exhibits a reproducible, isostructural spin transition characterized by a wide thermal hysteresis loop centred near room temperature (297 K) [15]. Here we show that this hysteretic region is associated with singular kinetic effects and exceptionally slow switching rates, whose origin can be traced back to the highly defective nature of the crystals.

\section{Experimental}

Single crystals of $\left[\mathrm{Fe}(1,6 \text {-naphthyridine })_{2}\left(\mathrm{Ag}(\mathrm{CN})_{2}\right)_{2}\right](\mathbf{1})$ were obtained by a slow diffusion method as previously described by some of us [15] (see also the Supporting Information, SI for the synthesis details). OM studies have been performed on several single crystals of 1 . The data shown in the manuscript refers to a crystal with dimensions of $44 \mu \mathrm{m} \times 39 \mu \mathrm{m} \times 10 \mu \mathrm{m}$. The crystal was placed on a glass slide and then enclosed in a temperature-controlled heating-cooling stage (THMS350V, Linkam Scientific Instruments). Before the experiments, the stage chamber was purged with dry nitrogen gas for ca. $3 \mathrm{~min}$ at $373 \mathrm{~K}$. Photographs were recorded in brightfield transmission mode using an Olympus BX51 upright microscope equipped with a $\times 50$ long working-distance objective (numerical aperture, NA $=0.5$ ) and a CCD camera (Clara, Andor Technology). Image acquisition was conducted either isothermally or at fixed heating/cooling 
rates (from 0.1 to $10 \mathrm{~K} \mathrm{~min}^{-1}$ ). A band-pass filter centred at $\lambda=532 \mathrm{~nm}$ was used to obtain a maximum optical contrast between the LS and HS phases. Image processing was performed using the ImageJ software.

\section{Results and discussion}

Recurrent pitfalls for spatiotemporal studies on single crystals is the sample-to-sample variation of the SCO properties due to different sample morphologies and microstructures and, for a given crystal, the progressive formation of defects and associated drift of the measured properties upon thermal cycling. These issues often make difficult the identification of the transition mechanism as well as the quantification of the associated kinetics. As a first step, we have thus carried out a series of preliminary measurements on different crystals of 1 . The most important findings are summarized in Figure 1. Overall, it appears that minor details of the thermal SCO curves differ both from crystal-to-crystal and from cycle-to-cycle, but the SCO behaviour appears sufficiently robust and reproducible to conduct more extensive investigations on single crystals of 1.

The hysteretic behaviour stems from the existence of a macroscopic free energy barrier (arising from cooperative intermolecular interactions), which defines a region of thermodynamic meta-stability between the LS and HS phases. Close enough to the equilibrium temperature, the macroscopic energy barrier is sufficiently high to make the lifetime of metastable states virtually infinite (compared to the experimental time scale), thus defining a quasi-static hysteretic region. However, as the lifetime of the metastable states progressively decreases as moving far from the equilibrium temperature, a kinetic dependence of the thermal hysteresis can be observed, characterized by an artificial increase of the hysteresis width. Indeed, numerous studies clearly demonstrated the strong dependence of the shape, sharpness, width and/or position of the hysteresis loop upon the temperature scan rate [16]. A spectacular example was reported on the mixed crystal series of the two-dimensional network $\left\{\left[\mathrm{Zn}_{1-\mathrm{x}} \mathrm{Fe} \mathrm{e}_{\mathrm{x}}(\mathrm{bbtr})_{3}\right]\left(\mathrm{ClO}_{4}\right)_{2}\right\}_{\infty}(\mathrm{bbtr}=1,4-$ di(1,2,3-triazol-1-yl)-butane). In this compound, temperature scan-rate studies have revealed that the apparent hysteresis loop emerging at low temperature (ca. 60-70 K) is of purely kinetic origin, owing to the slow HS-to-LS relaxation rate at this temperature [17]. Another remarkable example is that of the complex $\left[\mathrm{Fe}^{\prime \prime}(n \mathrm{Bu} \text {-im })_{3}(\right.$ tren $\left.)\right]\left(\mathrm{PF}_{6}\right)_{2}\left((n \mathrm{Bu} \text {-im })_{3}(\right.$ tren $)=$ tris $(\mathrm{n}$-butylimidazol(2-ethylamino))amine), which is characterised by an unusual increase of the thermal hysteresis width upon reducing the temperature scan rate (from $14-\mathrm{K}$-wide at $4 \mathrm{~K} \mathrm{~min}^{-1}$ to $41-\mathrm{K}$ wide at $0.1 \mathrm{~K} \mathrm{~min}^{-1}$ ) [18]. Such an effect is explained by the fact that two distinct LS phases, with 
different structural organizations, can be kinetically formed from the same HS phase, depending on the temperature sweep rate. Moreover, an important experimental fact is that the width of the thermal hysteresis may also simply depend on the thermal exchange coupling with the sample environment. As shown for single crystals of the SCO compound [Fe( $\mathrm{HB}(1,2,4$-triazol-1$\left.\mathrm{yl}_{3}\right)_{2}$, the transition temperatures, as well as the width of the thermal hysteresis loop, are independent of the temperature scan rate (up to at least $5 \mathrm{~K} \mathrm{~min}^{-1}$ ) at atmospheric pressure. However, by decreasing the air pressure down to $\mathrm{P}=0.1 \mathrm{mbar}$, noticeable changes were observed both on the thermal hysteresis width and on the switching kinetics of the crystal, due to the reduced efficiency of convective heat exchange between the sample and its environment [19]. The problem of heat exchange on the SCO hysteresis properties was also identified and investigated in single crystals of the SCO compound $\left[\left\{\mathrm{Fe}(\mathrm{NCSe})(\mathrm{py})_{2}\right\}_{2}(\mathrm{~m}-\mathrm{bpypz})\right](\mathrm{py}=$ pyridine and bpypz $=3,5$-bis(2-pyridyl)pyrazolate) [20].

Figure 2 depicts the typical spin-transition curves of a single crystal of 1 , obtained by varying the temperature scan rate from 0.1 to $10 \mathrm{~K} \mathrm{~min}^{-1}$. The values of the spin-transition temperatures in both heating and cooling modes $\left(\mathrm{T}_{1 / 2} \uparrow\right.$ and $\mathrm{T}_{1 / 2} \downarrow$, respectively) are summarized in Table $\mathrm{S} 1$ for the different scan rates used. As shown in Figure 2a, the LS-to-HS transition (heating mode) remains abrupt $(\Delta T<10 \mathrm{~K})$ whatever the scan rate. In contrast, the transition curves in the cooling mode exhibit a more noticeable scan-rate dependence. Indeed, while the HS-to-LS transition is rather abrupt at low scan rates $\left(\Delta \mathrm{T} \sim 10 \mathrm{~K}\right.$ at 0.1-1 $\left.\mathrm{K} \mathrm{min}^{-1}\right)$, much more gradual transitions are observed for high rates $\left(\Delta T \sim 30 \mathrm{~K}\right.$ at $\left.5-10 \mathrm{~K} \mathrm{~min}^{-1}\right)$. Thus, the increase of the temperature scan rate induces a pronounced enhancement of the asymmetry of the thermal hysteresis loop. This observation may suggest the existence of different driving/frictional forces for the spin transition in the two branches of the thermal hysteresis loop. As an example, the HS-to-LS and LS-to-HS transitions might involve distinct frictional forces due to the difference of stiffness between the LS and HS states. On the other hand, as depicted in Figure $2 b$, the shift of the transition temperatures is similar in the two branches: $T_{1 / 2} \downarrow$ (respectively $T_{1 / 2} \uparrow$ ) varies from $290.5 \mathrm{~K}$ (respectively $298 \mathrm{~K}$ ) to $280 \mathrm{~K}$ (respectively $308 \mathrm{~K}$ ) for scan rates of $0.1 \mathrm{~K} \mathrm{~min}^{-1}$ and $10 \mathrm{~K} \mathrm{~min}^{-1}$, respectively. As a result, the width of the thermal hysteresis goes from $7.5 \mathrm{~K}$ up to $27.5 \mathrm{~K}$ when increasing the scan rate from 0.1 to $10 \mathrm{~K} \mathrm{~min}^{-1}$.

Obviously, the symmetric widening of the hysteresis loop might be indicative of thermalization problems at high scan rates. However, our previous studies [19] using the same experimental setup discard this "extrinsic" origin of the hysteresis broadening in the present study. To 
investigate the plausible origin of such kinetic effects in $\mathbf{1}$, isothermal relaxation experiments were performed. The sample was cooled/heated at $1 \mathrm{~K} \mathrm{~min}^{-1}$ to various given temperatures within the hysteresis loop and the optical transmission across the crystal was monitored as a function of time at constant temperature (Figure 3). Further details on these isothermal experiments, including the relaxation curves, are provided in the SI. It is worth noting that a similar approach has been already used for other SCO systems to distinguish the quasi-static and "apparent" hysteresis loop [21]. It is important to stress, however, that in most cases the "apparent" hysteresis broadening has been observed at low temperatures (typically below ca. 100-120 K), mostly on the cooling branch, due to the slow spin-state interconversion rates at those temperatures.

In the case of 1, as depicted in Figure 3a for the cooling mode, no relaxation is observed (hour time scale) down to a temperature of $291.8 \mathrm{~K}$, this temperature thus defining the descending branch of the quasi-static hysteresis loop. For lower temperatures (for example, $291.0 \mathrm{~K}$ ) a slow relaxation is observed to the full LS state. One should note that the long relaxation times encountered here (up to 1 hour) definitely exclude the extrinsic origin (sic thermalization) of the observed phenomena. The same procedure in the heating mode allows to unveil a quasi-static 8K-wide hysteresis loop (Figure $3 \mathrm{~b}$ ). The width of this quasi-static hysteresis turns out to be approximately the same as that observed at the slowest scan rate $\left(\Delta T=7.5 \mathrm{~K}\right.$ at $\left.0.1 \mathrm{~K} \mathrm{~min}^{-1}\right)$. Such a quasi-static hysteresis implies the existence of a "real" thermodynamic bistability region (characterized by long-lived metastable states) near room temperature in this SCO material, which is an interesting asset for practical applications.

The spatiotemporal observation of the thermally induced spin transition in $\mathbf{1}$ also provides crucial information for the rationalization of the slow transition kinetics associated with the hysteresis. Figure 4 displays a set of selected images and movies showing the nucleation event(s) and the real-time evolution of the phase transition in the cooling and heating modes (see also the SI). These visual demonstrations reveal that the presence of numerous defects in the crystal undoubtedly impacts the spin-transition process. Indeed, the crystals of $\mathbf{1}$ appear systematically imperfect and can be better represented as a system consisting of many, small structural domains, which are more or less randomly oriented. This high degree of mosaicity was observed both via optical microscopy and single-crystal X-ray diffraction. ${ }^{\dagger}$ These different observations illustrate the so-called multi-scale causal chain of the SCO process proposed recently [22]. Indeed, it appears that microscopic details (defects, mosaicity) can have a huge impact on the 
macroscopic behaviour of the material, in particular here concerning the spin-state switching dynamics.

In the cooling regime, inhomogeneous nucleation of the LS phase simultaneously occurs in different places of the crystal, mostly near the edges, and several, non-consecutive phase boundaries propagate across the entire sample. On the contrary, in the heating mode, the nucleation of the HS phase always takes place in the middle of the crystal and a better-defined phase boundary propagates toward the edges of the crystal in a roughly circular manner. These features are found well reproducible for different crystals and for several consecutive thermal cycles. However, it should be noted that after typically more than 10 cycles some irreversible damage were observed in certain crystals (defects, cracks, etc.).

The singular spatiotemporal dynamics of the spin-state transformation in crystals of 1 can be qualitatively reproduced by kinetic Monte Carlo simulations, using a dynamic version of a spinphonon model. This class of models is known to be relevant for the theoretical investigations of the spatiotemporal development of the spin transition [23], since the lattice degree of freedom and the structural differences between the HS and LS phases are explicitly considered (see details in the SI). By introducing structural inhomogeneities within the system (i.e., a local tensile stress in the middle of the crystal lattice), we show that we are able to qualitatively reproduce some key features of the nucleation and growth phenomenon experimentally observed in crystals of 1. In particular, distinct nucleation sites for the LS (edges) and HS (middle of the crystal) phases as well as the existence of a quasi-circular phase-boundary propagation across the crystal can be depicted in the simulated images (Figure S6). This however raises questions about the possible origin of such a residual elastic stress gradient within the crystals of $\mathbf{1}$, especially in view of the synthesis method (slow diffusion), which might possibly lead to the build-up of stress inhomogeneities within the crystals.

A distinctive feature of the spin transition in crystals of $\mathbf{1}$ is the exceptionally slow kinetics of the growth process of the nucleated phase. Table 1 displays typical switching times $(\Delta t)$, temperature ranges $(\Delta T)$ and phase-boundary propagation velocities recorded in a crystal of 1 . Remarkably, the switching time at low scan rates $\left(0.1 \mathrm{~K} \mathrm{~min}^{-1}\right)$ is ca. $30-50 \mathrm{~min}$, which is ca. 4 orders of magnitude longer than the switching times (50-100 ms) previously reported for crystals of the SCO complex $\left[\mathrm{Fe}\left(\mathrm{HB}(1,2,4-\text { triazol-1-yl })_{3}\right)_{2}\right](2)$ [19] - in closely comparable experimental conditions. The difference of switching times clearly parallels the difference of phase-boundary velocities typically observed in the two materials. In crystals of $\mathbf{1}$, we observe propagation 
velocities in the range of $10^{-2}-10^{-1} \mu \mathrm{m} \mathrm{s}^{-1}$, down to $3 \mathrm{~nm} \mathrm{~s} \mathrm{~m}^{-1}$ (Table 1 ), whereas these velocity values fall in the $10^{2}-10^{3} \mu \mathrm{m} \mathrm{s}^{-1}$ range in crystals of 2 [19]. Previous studies of the nucleation and growth phenomenon in various SCO systems indicate that the average propagation velocity of the LS/HS phase boundary is typically $1-20 \mu \mathrm{m} \mathrm{s}^{-1}$, which is in between the two extreme cases represented by compounds 1 and 2 (Table S2) [10, 11].

Table 1. Switching temperature range $(\Delta \mathrm{T})$, switching time $(\Delta \mathrm{t})$ and typical phase-boundary propagation velocities (v) measured for a single crystal of $\mathbf{1}$ at various scan rates (dT/dt) in the heating and cooling modes.

\begin{tabular}{|c|c|c|c|c|}
\hline \multirow{4}{*}{ Cooling mode } & $\begin{array}{c}\mathbf{d T} / \mathbf{d t} \\
\left(\mathrm{K} \mathrm{min}^{-1}\right)\end{array}$ & $\begin{array}{c}\Delta \mathbf{T} \\
(\mathbf{K})\end{array}$ & $\begin{array}{c}\Delta \mathbf{t} \\
(\mathbf{m i n})\end{array}$ & $\begin{array}{c}\mathbf{V} \\
\left(\mathbf{n m ~ s}^{-1}\right)\end{array}$ \\
\cline { 2 - 5 } & 0.1 & 3 & 30 & 13 \\
\cline { 2 - 5 } & 0.5 & 9 & 18 & 23 \\
\cline { 2 - 5 } & 1.0 & 13 & 13 & 32 \\
\cline { 2 - 5 } & 5.0 & 35 & 7 & 160 \\
\cline { 2 - 5 } & 10 & 35 & 3.5 & 160 \\
\hline
\end{tabular}

\begin{tabular}{|c|c|c|c|c|}
\hline \multirow{4}{*}{ Heating mode } & $\begin{array}{c}\mathbf{d T} / \mathbf{d t} \\
\left(\mathrm{K} \mathrm{min}^{-1}\right)\end{array}$ & $\begin{array}{c}\Delta \mathbf{T} \\
(\mathrm{K})\end{array}$ & $\begin{array}{c}\Delta \mathbf{t} \\
(\mathbf{m i n})\end{array}$ & $\begin{array}{c}\mathbf{V} \\
\left(\mathbf{n m ~ s}^{-1}\right)\end{array}$ \\
\cline { 2 - 5 } & 0.1 & 5 & 50 & 3 \\
\cline { 2 - 5 } & 0.5 & 11 & 22 & 14 \\
\cline { 2 - 5 } & 1.0 & 11 & 11 & 24 \\
\cline { 2 - 5 } & 5.0 & 10 & 2 & 140 \\
\cline { 2 - 5 } & 10 & 10 & 1 & 240 \\
\hline
\end{tabular}

The exceptionally slow switching kinetics in crystals of 1 must certainly come from the existence of particularly strong resistive forces in this compound, whose effect is to hinder the phase-boundary motion. The main contribution of resistive forces commonly arises from the emergence of internal frictions and mechanical stresses during the phase-boundary propagation, due to the structural mismatch between the LS and HS phases. In addition, the role of crystal defects appears more dramatically in the present study. Cracks, failures and structural domain boundaries are numerous in crystals of 1 and obviously represent additional energy barriers for the propagation of the LS/HS phase boundary, making the domain growth mechanism a thermally-activated process at the origin of the "anomalous" hysteretic behaviour. The role of crystal defects is exemplified in Figure 4 (blue arrow), where a large failure is shown to open and close in a crystal during the spin transition.

\section{Conclusions}

In conclusion, we have performed an optical microscopy study of the thermally induced spin transition in single crystals of the SCO compound [Fe(1,6-naphthyridine $\left.)_{2}\left(\mathrm{Ag}(\mathrm{CN})_{2}\right)_{2}\right]$, including temperature scan-rate measurements, the assessment of the quasi-static hysteresis loop as well as the spatiotemporal investigation of the spin-state switching phenomenon. Despite the high transition temperatures (around room temperature), the thermal hysteresis loop is marked by pronounced kinetic effects, which appear to be related to the exceptionally slow growth rate of the nucleated phases. This slow switching process, which is a well-reproducible, general feature 
of this compound, is characterized by extremely slow propagation velocities of the LS/HS phase boundary (down to $3 \mathrm{~nm} \mathrm{~s}^{-1}$ ), the slowest known to date. Our OM studies reveal the critical influence of the unusually high number of microstructural defects in the crystals, which constitute a myriad of energy barriers to overcome, making the transition properties strongly dependent on the temperature scan rate. An important perspective of this work will be the investigation of size reduction effects on the switching kinetics. Indeed, as the number of structural domains decreases one can expect a significant acceleration of the switching rate approaching the intrinsic limit, represented by a perfect single crystal.

Notes

tFor the single crystal described in Chem. Commun. 2019, 55 (23), 3359-3362 [15] the mosaicity parameters are $2.54(\mathrm{e} 1=2.35, \mathrm{e} 2=1.10, \mathrm{e} 3=4.16)$ and $2.46(\mathrm{e} 1=1.82, \mathrm{e} 2=1.09, \mathrm{e} 3$ $=4.48$ ) degrees in the LS and HS forms, respectively (CrysAlisPro).

\section{Conflicts of interest}

The authors confirm that this article content has no conflict of interest.

\section{Acknowledgements}

This work was supported by H2020-MSCA-RISE-2016 Project 734322 and Projects 19BF03701M and 19BF037-04 of the Ministry of Education and Science of Ukraine. V. Hiiuk thanks Lozynskyj Foundation and Yuchymenko Family Endowment Fund for the financial support. Olesia I. Kucheriv is acknowledged for useful comments on the manuscript.

\section{References}

[1] P. Gütlich, H.A. Goodwin, Spin Crossover in Transition Metal Compounds I-III, SpringerVerlag, Berlin, Heidelberg, 2004.

[2] M.A. Halcrow, Spin-Crossover Materials: Properties and Applications, Wiley, 2013.

[3] A. Bousseksou, Spin crossover phenomenon/Phénomène de transition de spin, Vol. 21, Elsevier, Amsterdam, The Netherlands (2018), pp. 1055-1300.

[4] P. Gütlich, A. Hauser, H. Spiering, Thermal and Optical Switching of Iron(II) Complexes, Angew. Chem. Int. Ed. Engl. 33 (1994) 2024-2054.

https://doi.org/10.1002/anie.199420241

[5] O. Kahn, C. Jay Martinez, Spin-Transition Polymers: From Molecular Materials Toward Memory Devices, Science 279 (1998) 44-48. 
[6] C. Arnaud, T. Forestier, N. Daro, E. Freysz, J.-F. Létard, G. Pauliat, G. Roosen, Observation of an asymmetry in the thermal hysteresis loop at the scale of a single spin-crossover particle, Chem. Phys. Lett. 470 (2009) 131-135.

https://doi.org/10.1016/j.cplett.2009.01.014.

[7] C.M. Quintero, I.A. Gural'skiy, L. Salmon, G. Molnár, C. Bergaud, A. Bousseksou, Soft lithographic patterning of spin crossover complexes. Part 1: fluorescent detection of the spin transition in single nano-objects, J. Mater. Chem. 22 (2012) 3745. https://doi.org/10.1039/c2jm15662h.

[8] S. Liu, K. Zhou, T. Yuan, W. Lei, H.-Y. Chen, X. Wang, W. Wang, Imaging the Thermal Hysteresis of Single Spin-Crossover Nanoparticles, J. Am. Chem. Soc. 142 (2020) 1585215859. https://doi.org/10.1021/jacs.0c05951.

[9] J. Jeftic, F. Varret, A. Hauser, O. Roubeau, M. Matsarski, J.-P. Rivera, Patterns During Photoexcitation and High-Spin $\rightarrow$ Low-Spin Relaxation in $\left[\mathrm{Fe}(\mathrm{ptz})_{6}\right]\left(\mathrm{BF}_{4}\right)_{2}$ Spin Transition Crystal, Mol. Cryst. Liq. Cryst. Sci. Technol. Sect. A. Mol. Cryst. Liq. Cryst. 335 (1999) 511520. https://doi.org/10.1080/10587259908028892.

[10] F. Varret, C. Chong, A. Slimani, D. Garrot, Y. Garcia and A. D. Naik, Real-Time Observation of Spin-Transitions by Optical Microscopy, Spin-Crossover Materials: Properties and Applications, John Wiley \& Sons Ltd., New York, 2013, pp. 425-441.

[11] K. Ridier, G. Molnár, L. Salmon, W. Nicolazzi, A. Bousseksou, Hysteresis, nucleation and growth phenomena in spin-crossover solids, Solid State Sci. 74 (2017) A1-A22. https://doi.org/10.1016/j.solidstatesciences.2017.10.014.

[12] H. Fourati, E. Milin, A. Slimani, G. Chastanet, Y. Abid, S. Triki, K. Boukheddaden, Interplay between a crystal's shape and spatiotemporal dynamics in a spin transition material, Phys. Chem. Chem. Phys. 20 (2018) 10142-10154. https://doi.org/10.1039/C8CP00868J.

[13] R. Traiche, H. Oubouchou, M. Zergoug, K. Boukheddaden, Spatio-temporal aspects of the domain propagation in a spin-crossover lattice with defect, Phys. B Condens. Matter. 516 (2017) 77-84. https://doi.org/10.1016/j.physb.2017.04.026.

[14] S. Bedoui, M. Lopes, S. Zheng, S. Bonnet, G. Molnár, A. Bousseksou, Laser-Induced Artificial Defects (LIADs): Towards the Control of the Spatiotemporal Dynamics in Spin Transition Materials, Adv. Mater. 24 (2012) 2475-2478. https://doi.org/10.1002/adma.201200386.

[15] V.M. Hiiuk, S. Shova, A. Rotaru, V. Ksenofontov, I.O. Fritsky, I.A. Gural'skiy, Room temperature hysteretic spin crossover in a new cyanoheterometallic framework, Chem. Commun. 55 (2019) 3359-3362. https://doi.org/10.1039/C8CC10260K.

[16] S. Brooker, Spin crossover with thermal hysteresis: practicalities and lessons learnt, Chem. Soc. Rev. 44 (2015) 2880-2892. https://doi.org/10.1039/C4CS00376D.

[17] P. Chakraborty, C. Enachescu, C. Walder, R. Bronisz, A. Hauser, Thermal and Light- 
Induced Spin Switching Dynamics in the 2D Coordination Network of $\left\{\left[\mathrm{Zn}_{1-}\right.\right.$ $\left.\left.{ }_{x} \mathrm{Fe}_{x}(\text { bbtr })_{3}\right]\left(\mathrm{ClO}_{4}\right)_{2}\right\}_{\infty}$ : The Role of Cooperative Effects, Inorg. Chem. 51 (2012) 97149722. https://doi.org/10.1021/ic301006c.

[18] M. Seredyuk, M.C. Muñoz, M. Castro, T. Romero-Morcillo, A.B. Gaspar, J.A. Real, Unprecedented Multi-Stable Spin Crossover Molecular Material with Two Thermal Memory Channels, Chem. - A Eur. J. 19 (2013) 6591-6596.

https://doi.org/10.1002/chem.201300394.

[19] K. Ridier, S. Rat, L. Salmon, W. Nicolazzi, G. Molnár, A. Bousseksou, Scan-rate and vacuum pressure dependence of the nucleation and growth dynamics in a spin-crossover single crystal: the role of latent heat, Phys. Chem. Chem. Phys. 20 (2018) 9139-9145. https://doi.org/10.1039/C7CP08522B.

[20] R. Traiche, M. Sy, H. Oubouchou, G. Bouchez, F. Varret, K. Boukheddaden, Spatiotemporal Observation and Modeling of Remarkable Temperature Scan Rate Effects on the Thermal Hysteresis in a Spin-Crossover Single Crystal, J. Phys. Chem. C 121 (2017) 11700-11708. https://doi.org/10.1021/acs.jpcc.7b02816.

[21] A.F. Stassen, O. Roubeau, I. Ferrero Gramage, J. Linarès, F. Varret, I. Mutikainen, U. Turpeinen, J.G. Haasnoot, J. Reedijk, Physical properties of the spin-crossover compound hexakis(1-methyltetrazole- $\mathrm{N}^{4}$ )iron(II) triflate, steady state and relaxation studies, $\mathrm{X}$-ray structure of the isomorphic Ni(II) compound, Polyhedron 20 (2001) 1699-1707. https://doi.org/10.1016/S0277-5387(01)00674-X.

[22] P. Guionneau, M. Marchivie, G. Chastanet, Multiscale Approach of Spin Crossover Materials: A Concept Mixing Russian Dolls and Domino Effects, Chem. - A Eur. J. 27 (2021) 1483-1486. https://doi.org/10.1002/chem.202002699.

[23] C. Enachescu, W. Nicolazzi, Elastic models, lattice dynamics and finite size effects in molecular spin crossover systems, C. R. Chimie 21 (2018) 1179-1195. https://doi.org/10.1016/j.crci.2018.02.004. 
a)

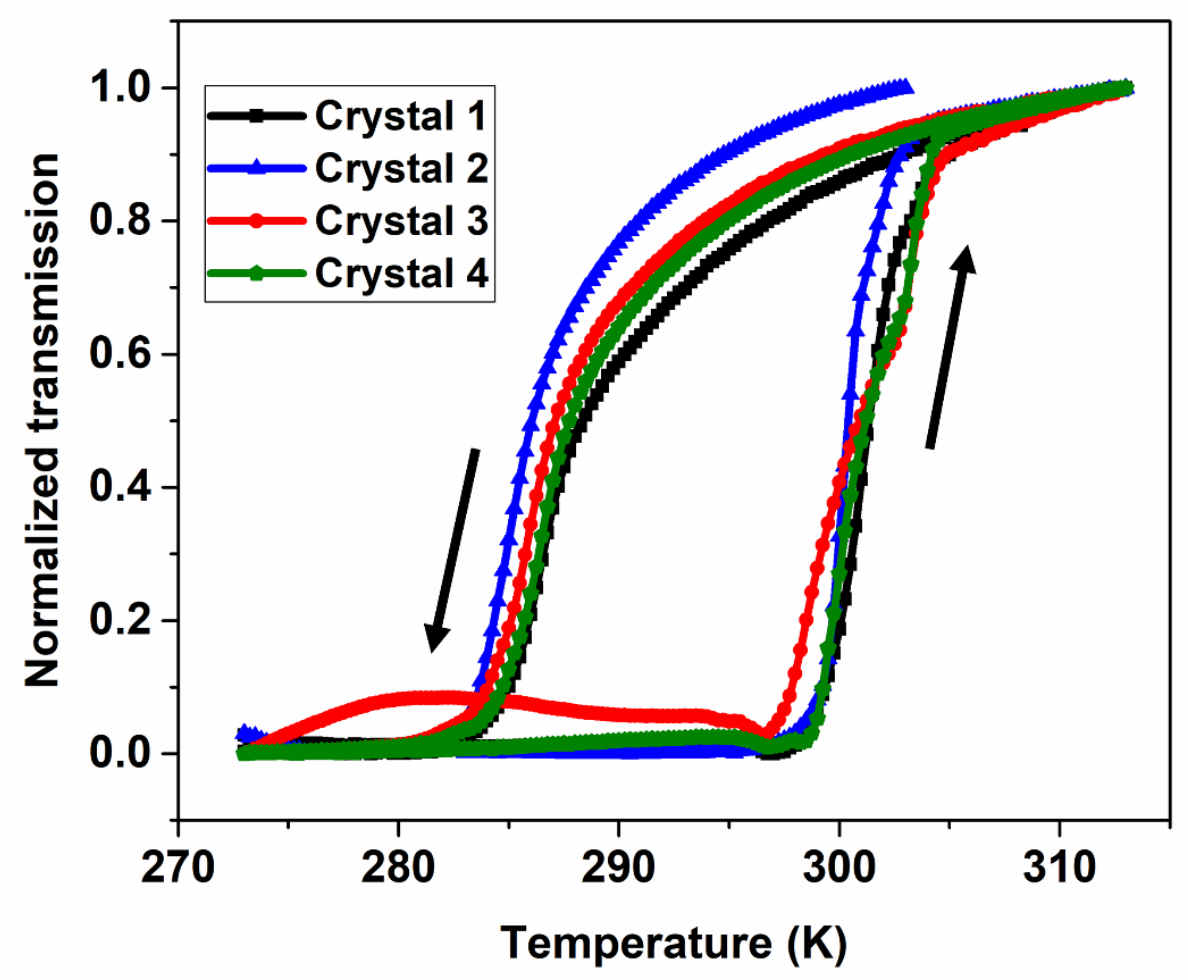

b)

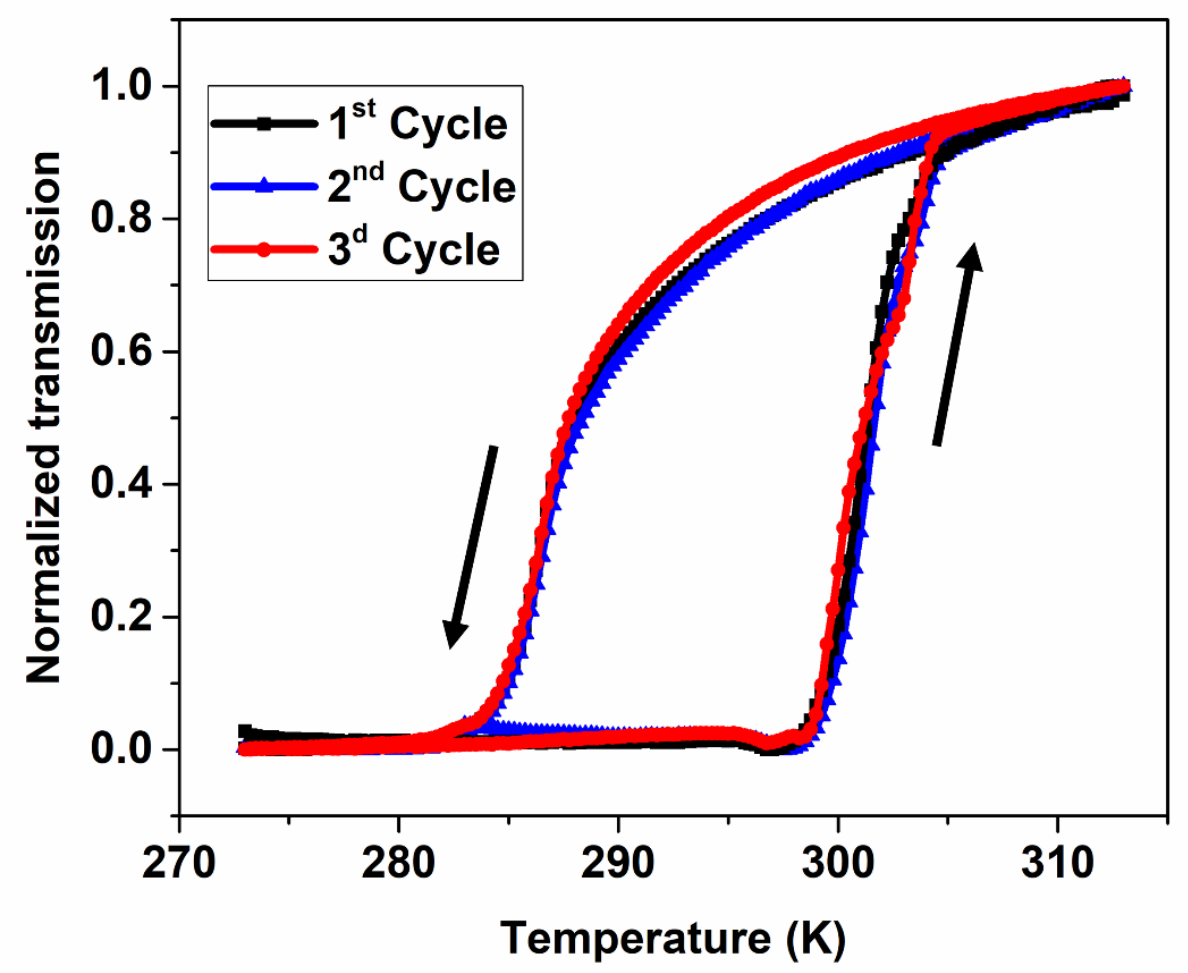

Figure 1. (a) Reproducibility of the SCO in four different single crystals of 1. (b) Reproducibility of the SCO in a single crystal of 1 for three subsequent thermal cycles. All data were acquired at $1 \mathrm{~K}$ $\min ^{-1}$. Heating and cooling are indicated by arrows. 
a)

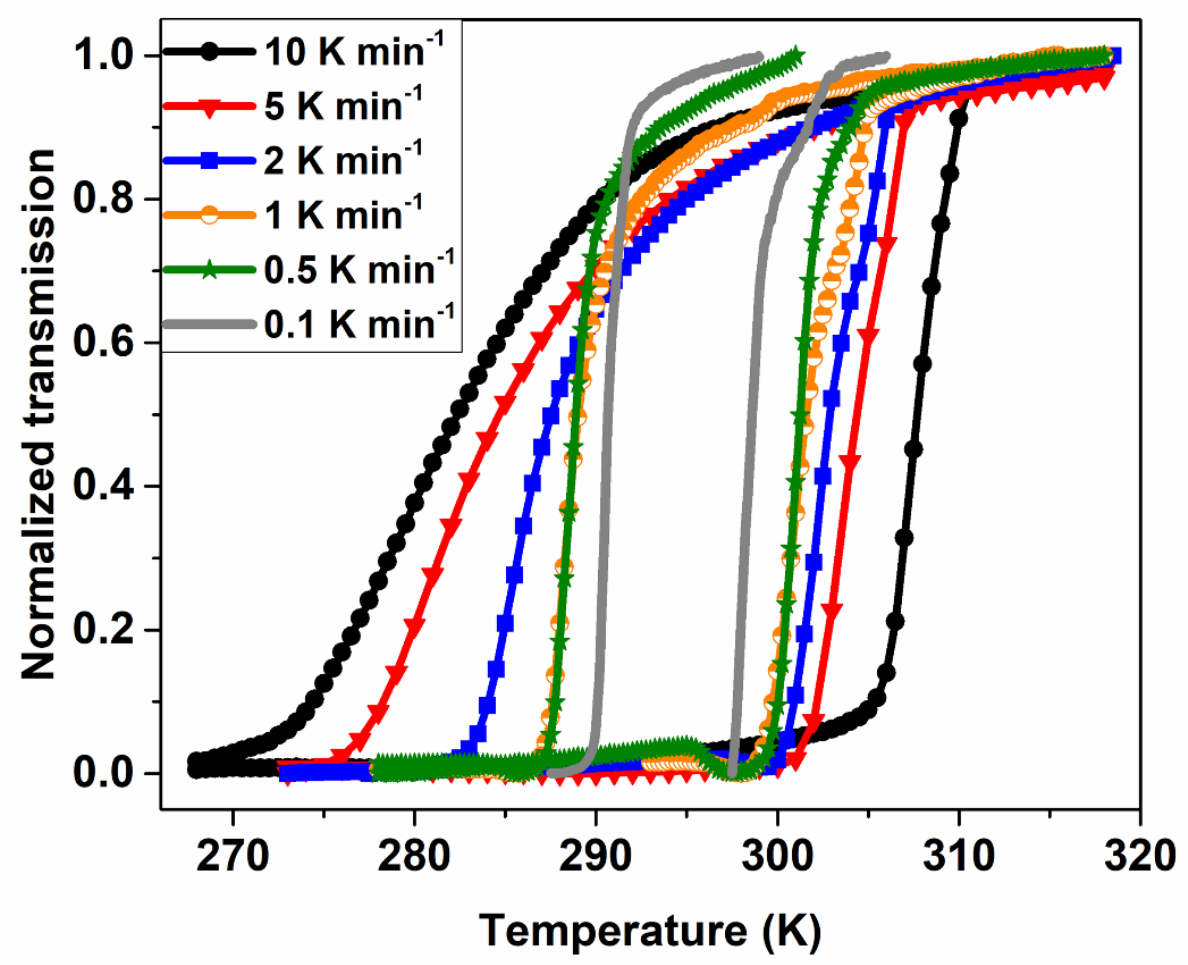

b)

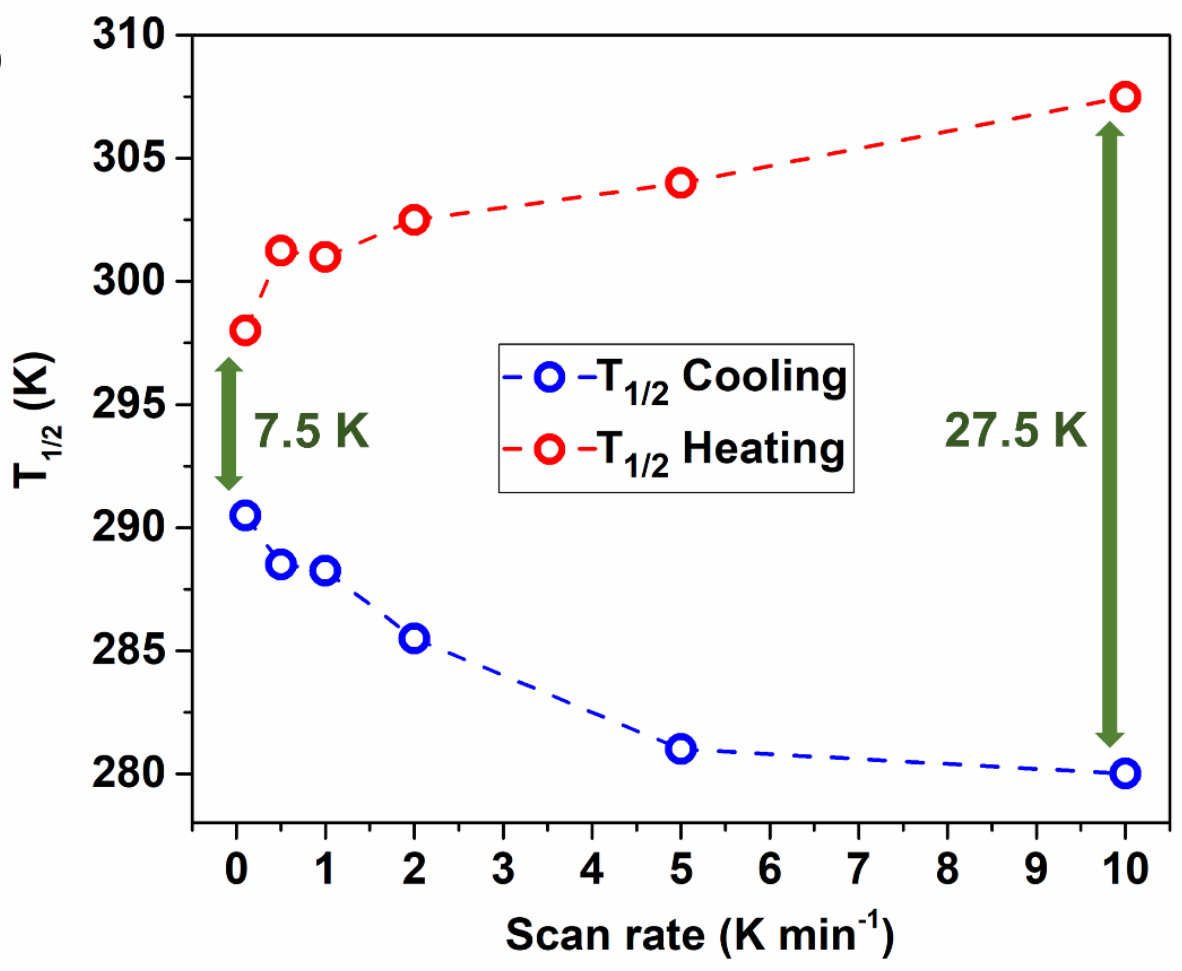

Figure 2. (a) Temperature scan-rate study $\left(0.1-10 \mathrm{~K} \mathrm{~min}^{-1}\right)$ of the spin transition (normalized optical transmission vs. temperature) in a single crystal of 1. (b) Scan-rate dependence of the spin-transition temperatures in the heating and cooling modes. 

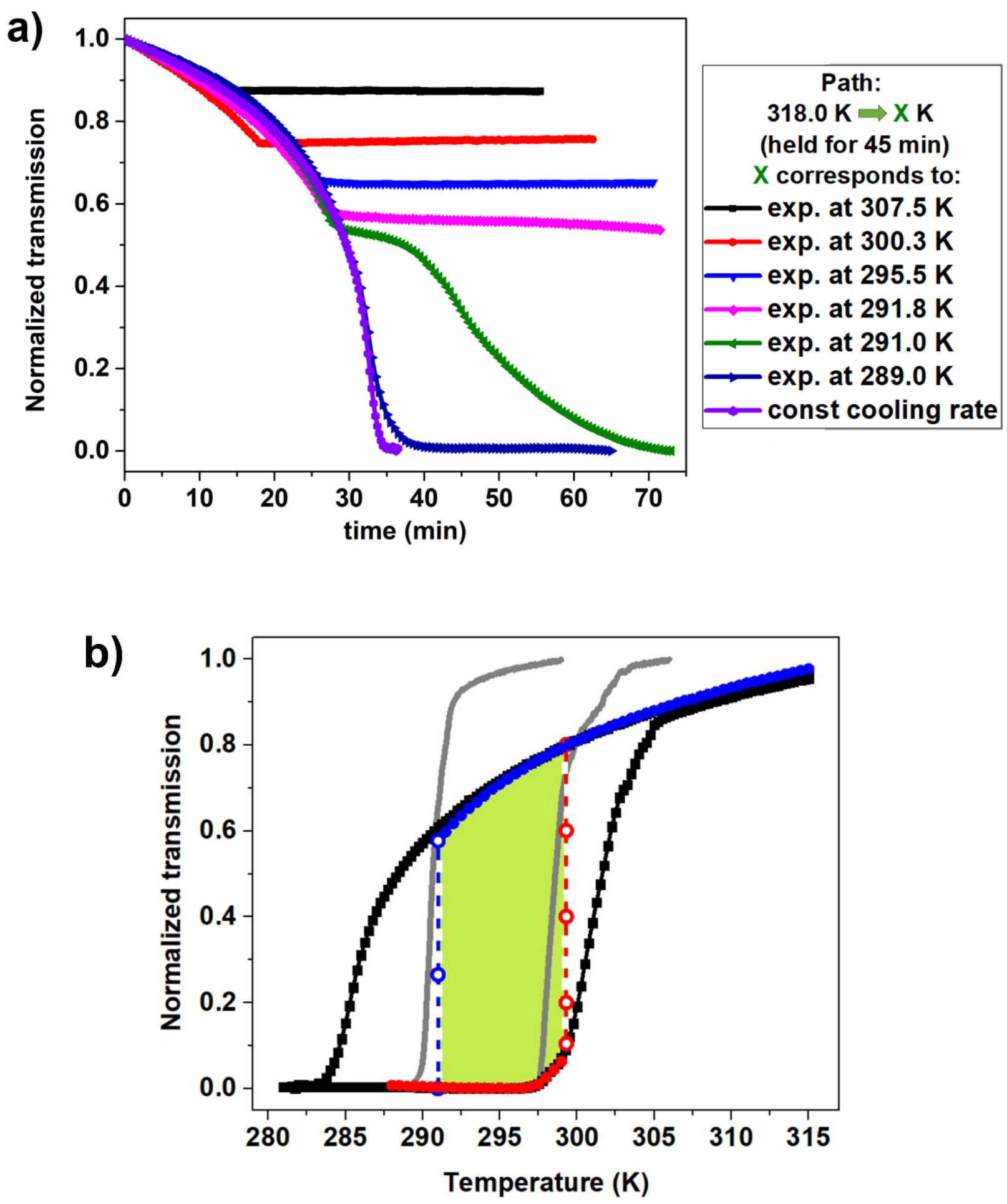

Figure 3. (a) Isothermal HS $\rightarrow$ LS relaxation measurements on a crystal of 1 at selected temperatures. A reference curve acquired at constant cooling rate $\left(1 \mathrm{~K} \mathrm{~min}^{-1}\right)$ is included (violet line). (b) Quasi-static hysteresis loop (green region) extracted from the isothermal relaxation experiments. Two reference curves, recorded in heating and cooling modes at scan rates of $1 \mathrm{~K}$ $\min ^{-1}$ (black line) and $0.1 \mathrm{~K} \mathrm{~min}^{-1}$ (grey line), are also shown. 


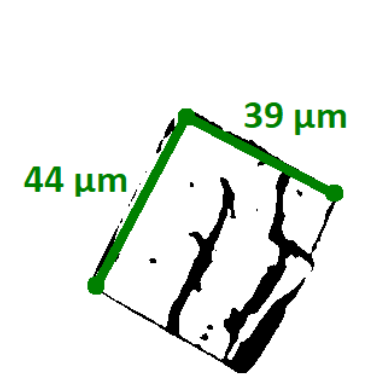

HS

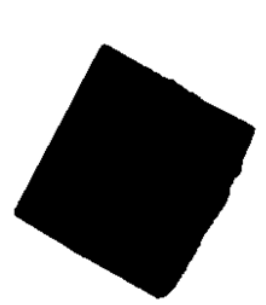

LS

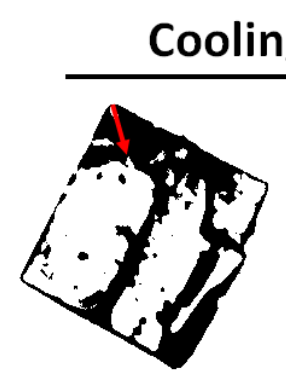

HS/LS = 3:1

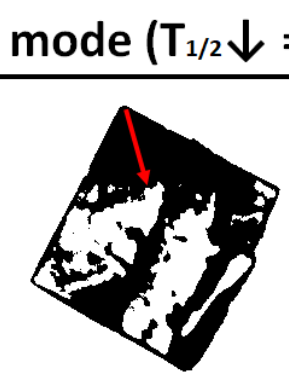

$H S / L S=1: 1$

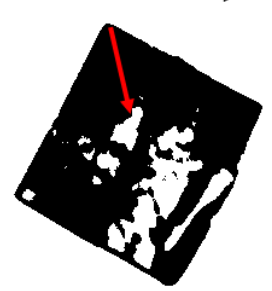

HS/LS = 1:3

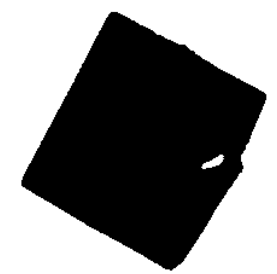

LS

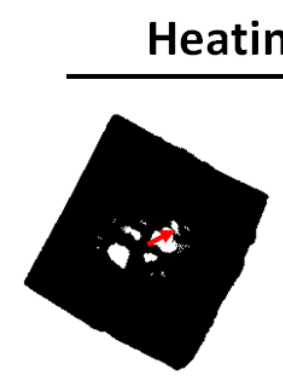

LS/HS = 3:1

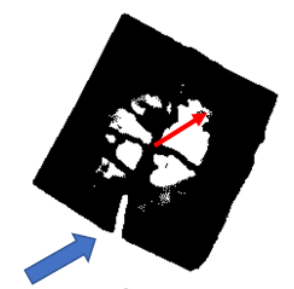

LS/HS = 1:1

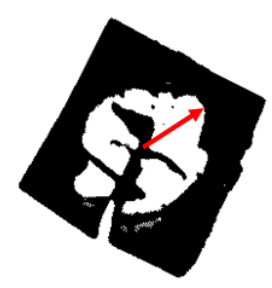

LS/HS = 1:3

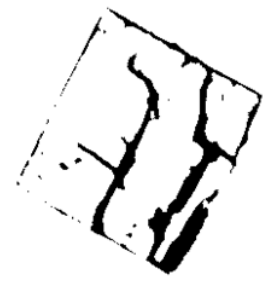

HS

Figure 4. Snapshots of the spin-state transformation of a crystal of $\mathbf{1}$ in the cooling and heating modes at $0.1 \mathrm{~K} \mathrm{~min}^{-1}$. Red arrows indicate the propagation of phase boundaries. The blue arrow shows the opening and closing of a failure in the course of the phase transition. 